\title{
¿Validation of a Prototype Global 4D-Var Data Assimilation System for the MPAS-Atmosphere Model
}

\author{
XiaOXu Tian ${ }^{\mathrm{a}}$ AND XiaOlei ZOU $^{\mathrm{b}}$ \\ ${ }^{a}$ Department of Atmospheric and Oceanic Science, University of Maryland, College Park, College Park, Maryland \\ ${ }^{\mathrm{b}}$ Nanjing University of Information Science and Technology, Nanjing, China
}

(Manuscript received 15 December 2020, in final form 28 May 2021)

\begin{abstract}
A four-dimensional variational (4D-Var) data assimilation (DA) system is developed for the global nonhydrostatic atmospheric dynamical core of the Model for Prediction Across Scales (MPAS). The nonlinear forward and adjoint models of the MPAS-Atmosphere dynamic core are included in a Python-driven structure to formulate a continuous 4D-Var DA system, shown to effectively minimize the cost function that measures the distances between the nonlinear model simulations and observations. In this study, three idealized experiments with a 6-h assimilation window are conducted to validate and demonstrate the numerical feasibilities of the 4D-Var DA system for both uniform- and variable-resolution meshes. In the first experiment, only a single point observation is assimilated. The resulting solution shows that the analysis increments have highly flow-dependent features. The observations in the second experiment are all model prognostic variables that span the entire global domain, the purpose of which is to check how well the initial conditions $6 \mathrm{~h}$ prior to the observations can be reversely inferred. The differences between the analysis and the referenced "truth" are significantly smaller than those calculated with the first guess. The third experiment assimilates the mass field only, i.e., potential temperatures in the case of MPAS-Atmosphere, and examines the impacts on the wind field and the mass field under initial conditions. Both the wind vectors and potential temperatures in the analysis agree more with the referenced "truth" than the first guess because the adjustments made to the initial conditions are dynamically consistent in the 4D-Var system.
\end{abstract}

KEYWORDS: Data assimilation; Nonhydrostatic models; Numerical weather prediction/forecasting; Variational analysis

\section{Introduction}

Numerically solving the atmospheric governing equations has been heavily relied upon to make accurate weather forecasts. Lorenz (1975) pointed out that weather forecast uncertainties generally come from errors in the initial conditions and numerical forecast models. With a given model, predicting the weather is largely an initial-value problem where future atmospheric states are predicted from the current atmospheric state, i.e., the initial conditions (Zou et al. 2001). The accurate estimation of initial conditions is thus critical for making reliable weather forecasts. The technique of four-dimensional variational (4D-Var) data assimilation (DA) has been one of the most sought-after methods in the field of atmospheric science because it offers a rigorous mathematical foundation for obtaining the optimal estimate of model initial conditions based on prior knowledge of the atmospheric state and observed features (Lewis and Derber 1985; Le Dimet and Talagrand 1986; Navon et al. 1992; Zou et al. 1995). The use of adjoint models in the 4D-Var DA system brings about the advantages of 4D-Var, including 1) efficient and accurate calculations of the gradients of the cost function that have the time of the observations accounted for, 2) implicit evolutions of the forecast error covariances following the nonlinear flow

¿ Denotes content that is immediately available upon publication as open access.

Corresponding author: Xiaoxu Tian, xtian15@terpmail.umd.edu trajectories, and 3) dynamical consistency in the analyzed solutions (Navon et al. 1992; Huang et al. 2009). Given these strengths of the 4D-Var technique, numerous previous studies and operational centers have sought to adopt 4D-Var into their most sophisticated weather forecast applications. Zou et al. (1995) and Zou et al. (1997) developed the adjoint model of the fifth-generation Pennsylvania State University-National Center for Atmospheric Research (NCAR) Mesoscale Model and formulated a 4D-Var regional DA system. Zou et al. (2001) proposed a global 4D-Var DA system based on the National Centers for Environmental Prediction global spectral model. The 4D-Var DA system in the Integrated Forecasting System at the European Centre for Medium-Range Weather Forecasts (ECMWF) marked the first successful operational implementation (Courtier et al. 1994). Other operational centers that adopted 4D-Var for operational applications include, but are not limited to, Météo-France (Gauthier and Thepaut 2001), the Met Office (Lorenc and Rawlins 2005), the Swedish Meteorological and Hydrological Institute (Gustafsson 2006; Gustafsson et al. 2012), and the China Meteorological Administration (Zhang et al. 2019).

The Model for Prediction Across Scales-Atmosphere (MPAS-A) is a global modeling framework released by NCAR (Skamarock et al. 2012; Park et al. 2013). Over the global domain, the MPAS-A employs finite-volume irregular centroidal Voronoi meshes on a staggered C-grid (Fig. 1) that allows for numerically solving atmospheric governing equations at both uniform (Fig. 1a) and smoothly variable (Fig. 1b) resolutions. Hagos et al. (2013) demonstrated the advantages of the smoothly variable resolution over the previous abruptly 


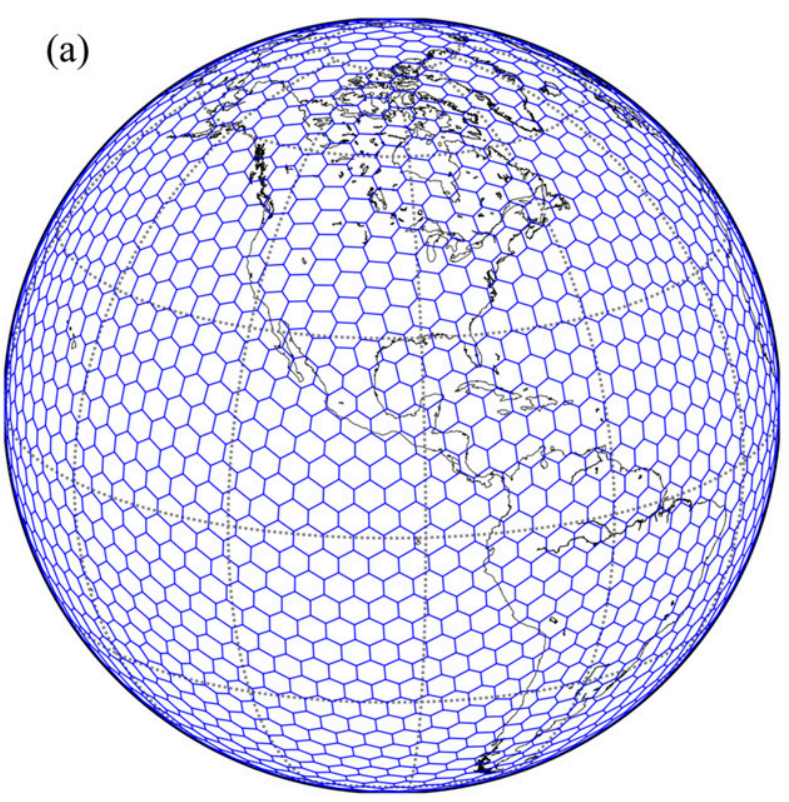

(b)

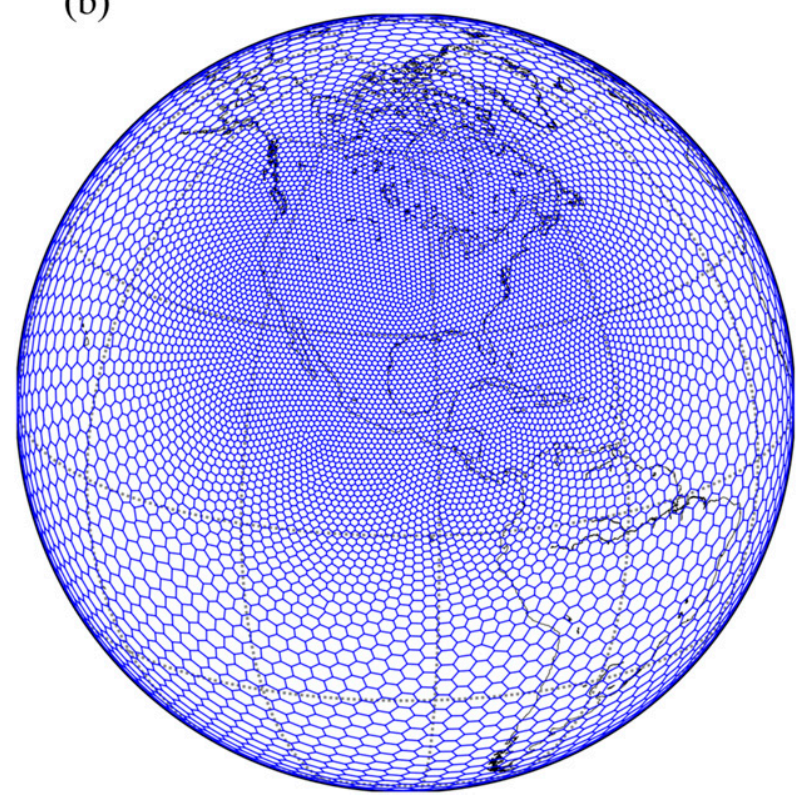

FIG. 1. Spatial distributions of the spherical centroidal Voronoi tessellation meshes with (a) 2562 grid cells at a quasi-uniform resolution of $480 \mathrm{~km}$ and (b) 10242 grid cells at resolutions ranging from 100 to $400 \mathrm{~km}$ over the globe in the MPAS-Atmosphere. The center of the area with a refined resolution in (b) is located at $35^{\circ} \mathrm{N}, 95^{\circ} \mathrm{W}$.

changing resolution in the nesting approach of the Weather Research and Forecasting (WRF) Model, which effectively avoided the erroneous wave signals generated in WRF simulations with a two-way nesting configuration. The option of doing simulations with variable resolutions implies that highresolution regional climatological simulations may be achieved at an economical computational cost without depending on any lateral boundary conditions (Michaelis et al. 2019).

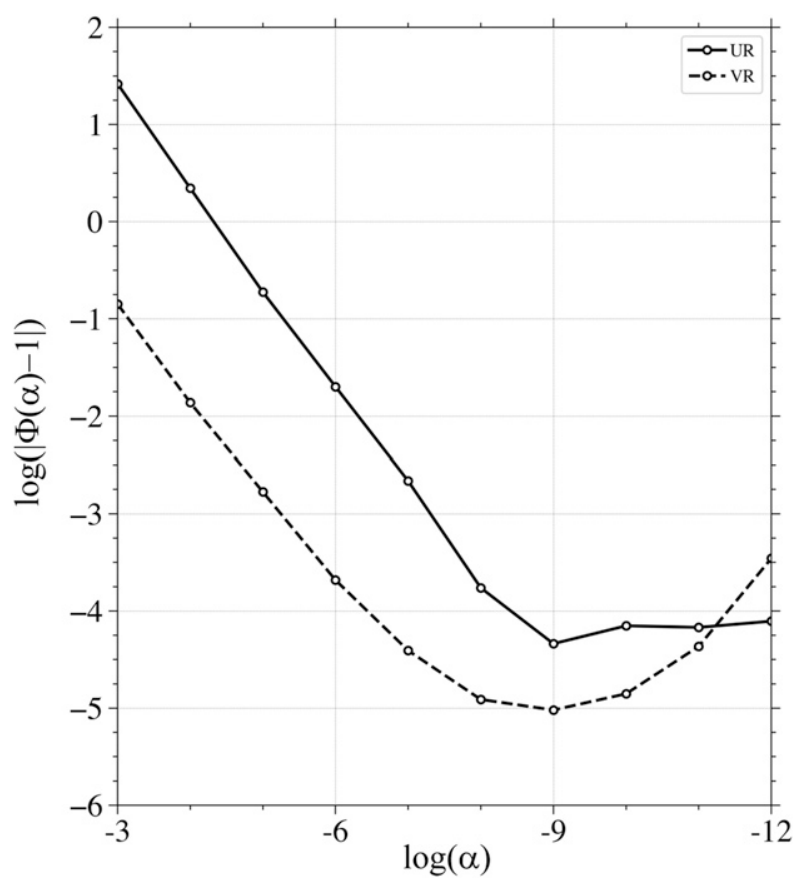

FIG. 2. Variations in the gradient-check results for the uniform resolution (solid curve) and variable-resolution (dashed curve) meshes following Eq. (6), i.e., $\log (|\Phi(\alpha)-1|)$, as a function of the $\log$ of the scaling factor $\alpha$.

Tian and Zou (2020) documented the development and verification of the tangent linear (TL) and adjoint (AD) models of the MPAS-A and showed their usefulness in a sensitivity analysis using the MPAS AD model. Note that the TL/AD models of MPAS-A, like the original nonlinear forecast model, were developed in FORTRAN for its fast computational speed, while the top-layer structure was written in Python for its ease and flexibility in input/output, controlling simulation flows, and manipulating matrices (Lin 2012). In this study, the dynamic core of the MPAS-A nonlinear forecast model in Skamarock et al. (2012), i.e., the nonlinear forward model, and the AD model in Tian and Zou (2020) are used to formulate a global continuous 4D-Var DA system. No physics is included for the time being because the system is still under development. Similar to the structure in Tian and Zou (2020), the heavy-duty nonlinear forward and adjoint calculation processes are maintained in FORTRAN because of its efficiency, and the 4D-Var framework is coded in Python for its convenience. With such a framework, obtaining the gradients of the cost function and minimizations are still efficient, while the DA system's overall Python coordinating structure is easily readable and adjustable. The FORTRAN codes are compiled with a Python utility f2py to build the interfaces between the two programming languages. With such interfaces, the computation results from the components of the nonlinear forward, adjoint, and minimization are communicated directly in memory. Section 2 of this study briefly introduces the TL/AD models of the MPAS-A and the formulation of a 4D-Var DA system. Section 3 describes the configurations 


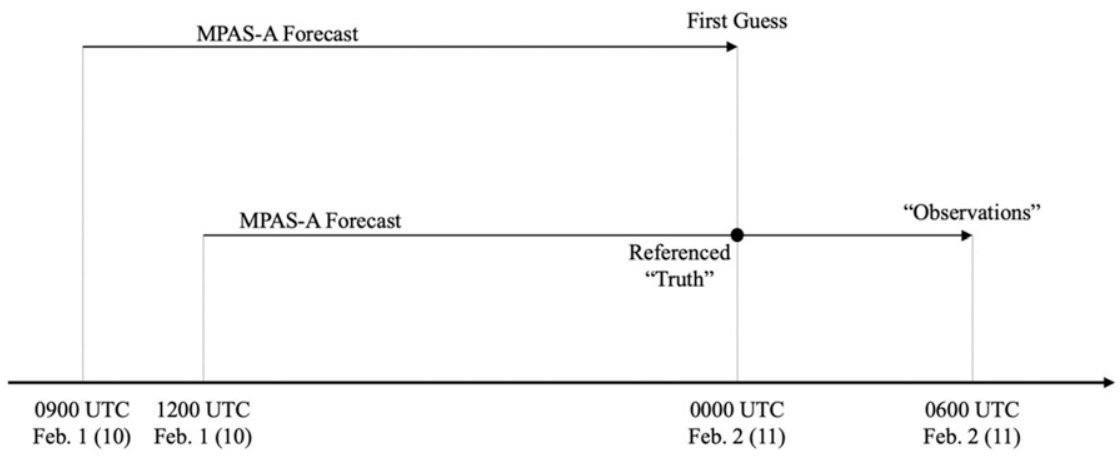

FIG. 3. A schematic illustration of the generation of the first guess, "observations," and referenced "truth" in the three idealized experiments.

of three idealized experiments that are done to validate and demonstrate the MPAS-A 4D-Var DA system's performance. Numerical results of the three experiments for both the uniform- and variable-resolution meshes are shown and described in section 4 . Section 5 gives a summary and conclusions.

\section{MPAS-A 4D-Var DA system}

\section{a. Development of the MPAS-A TL/AD models}

The nonhydrostatic MPAS-A forward model can be expressed as

$$
\mathbf{x}\left(t_{r}\right)=\mathcal{M}\left[\mathbf{x}\left(t_{0}\right)\right],
$$

where $\mathbf{x}$ denotes the five model prognostic variables, i.e., wind component normal to the cell edges $u$, vertical velocity $w$, air density $\rho$, potential temperature $\theta$, and water vapor mixing ratio $q_{v}$. The model takes the initial condition $\mathbf{x}\left(t_{0}\right)$ at the starting time $t_{0}$ as input and numerically solves the nonhydrostatic atmospheric dynamical governing equations described by Skamarock et al. (2012) to obtain the state variables $\mathbf{x}\left(t_{r}\right)$ at forecast time $t_{r}$. The term $\mathcal{M}$, in this case, is called the nonlinear MPAS-A forward operator. The TL model of the MPAS-A is developed following the relationship:

$$
\Delta \mathbf{x}\left(t_{r}\right)=\mathbf{M}\left[\mathbf{x}\left(t_{0}\right)\right] \Delta \mathbf{x}\left(t_{0}\right)=\frac{\partial \mathcal{M}\left[\mathbf{x}\left(t_{0}\right)\right]}{\partial \mathbf{x}} \Delta \mathbf{x}\left(t_{0}\right),
$$

where the symbol $\Delta$ represents perturbations to the nonlinear prognostic variables x (Zou et al. 1997; Tian and Zou 2020). The tangent linear operator $\mathbf{M}$ is the partial derivative of $\mathcal{M}$ with respect to each of the prognostic and diagnostic variables in the nonlinear forward MPAS-A. The TL model takes perturbations of small magnitude $\Delta \mathbf{x}\left(t_{0}\right)$ at time $t_{0}$ as inputs and predicts the perturbations $\Delta \mathbf{x}\left(t_{r}\right)$ at the forecast $t_{r}$ evolved along the nonlinear state trajectory. The adjoint model of MPAS-A, $\mathbf{M}^{\mathrm{T}}$, is essentially the transpose of the MPAS-A TL model $\mathbf{M}$, which can then be written as

$$
\Delta \hat{\mathbf{x}}\left(t_{0}\right)=\mathbf{M}^{\mathrm{T}}\left[\mathbf{x}\left(t_{0}\right)\right] \Delta \hat{\mathbf{x}}\left(t_{r}\right) .
$$

In Eq. (3), $\Delta \hat{\mathbf{x}}$ denotes the $\mathrm{AD}$ variables. In contrast to the nonlinear forward or TL models, the AD model integration starts at the forecast time $t_{r}$ and ends at time $t_{0}$. During the backward integrations of the AD model, the nonlinear forward trajectory of the prognostic variables is necessary for calculating the $\mathrm{AD}$ variables. Given the same simulation time window, the computational cost of the MPAS-A AD model is about 4.5 times that of the nonlinear forward model (Tian and Zou 2020). At this stage, the models compute in a serial fashion.

\section{b. Variational analysis}

A DA problem is to find a model trajectory that fits both the observations available at different time moments within the assimilation window $\left[t_{0}, t_{r}\right]$ and the most recent forecast representing the atmospheric state at $t_{0}$, i.e., the first guess. A scalar function of the model prognostic variables $\mathbf{x}$ is defined as

$$
\begin{aligned}
J= & J_{b}+J_{o} \\
= & \frac{1}{2}\left(\mathbf{x}_{0}-\mathbf{x}_{b}\right)^{\mathrm{T}} \mathbf{B}^{-1}\left(\mathbf{x}_{0}-\mathbf{x}_{b}\right) \\
& +\frac{1}{2} \sum_{r=0}^{N}\left[H_{r}\left(\mathbf{x}_{r}\right)-\mathbf{y}_{r}\right]^{\mathrm{T}} \mathbf{O}_{r}^{-1}\left[H_{r}\left(\mathbf{x}_{r}\right)-\mathbf{y}_{r}\right],
\end{aligned}
$$

where $\mathbf{x}_{0}$ is the analysis vector at time $t_{0}$ to be solved, $\mathbf{x}_{b}$ is the background field, $\mathbf{x}_{r}$ is the model state at $t_{r}$ evolved from $\mathbf{x}_{0}, \mathbf{y}_{r}$ represents any observed quantity, and $H_{r}$ is the observation operator that transforms $\mathbf{x}_{r}$ to the observation space at the time of the observations (Zou et al. 1995, 1997). The matrices B and $\mathbf{O}$ are the background and observation error covariance matrices, respectively. The first term $J_{b}$ measures the distance between the first-guess background and the analysis vector, and the second term $J_{o}$ is the summation of the distances between the observations and the analysis vector evolved to the observation time by the nonlinear forecast model. Both are then weighted by their respective error covariance matrices.

The gradient of the cost function with respect to the analysis vector $\mathbf{x}_{0}$ can be calculated as

$$
\nabla_{\mathbf{x}_{0}} J=\mathbf{B}^{-1}\left(\mathbf{x}_{0}-\mathbf{x}_{b}\right)+\sum_{r=0}^{N} \mathbf{M}_{r}^{\mathrm{T}} \mathbf{H}_{r}^{\mathrm{T}} \mathbf{O}_{r}^{-1}\left[H_{r}\left(\mathbf{x}_{r}\right)-\mathbf{y}_{r}\right]
$$

where $\mathbf{M}_{r}^{\mathrm{T}}$ is the AD model of MPAS-A, and $\mathbf{H}_{r}^{\mathrm{T}}$ is the AD of the observation operator $H_{r}$ (Zou et al. 1997; Tian and Zou 2019). 

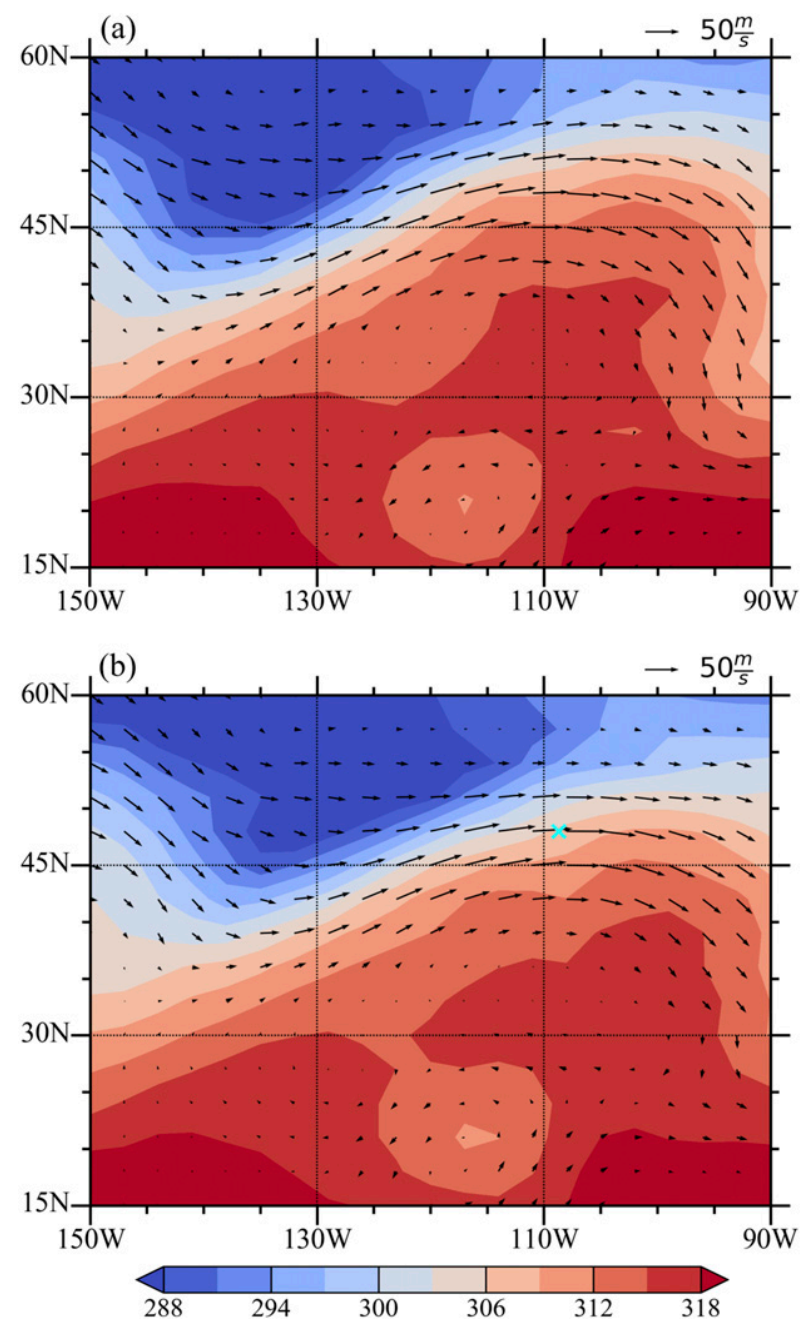

FIG. 4. Spatial distributions of potential temperature (shaded; K) and wind vectors at $500 \mathrm{hPa}$ in the forecast valid for (a) $0000 \mathrm{UTC}$ (the first guess) and (b) 0600 UTC ("observations") 2 Feb 2020. The first guess is the forecast by MPAS taking ERA5 at 0900 UTC 1 Feb 2020 as the initial conditions and the "observations" taking ERA5 at 1200 UTC 1 Feb 2020 as the initial conditions. The location of the single observation assimilated is indicated by the cyan cross in (b).

In Eq. (5), the discrepancy between the transformed model state vector and observations at time $t_{r},\left[H_{r}\left(\mathbf{x}_{r}\right)-\mathbf{y}_{r}\right]$, serves as the observational forcing and is weighted by the observation error covariances $\mathbf{O}_{r}^{-1}$. The forcing term is then transformed back to the model grid space by the AD of the observation operator $\mathbf{H}_{r}^{\mathrm{T}}$ and input into the MPAS-A AD model that will integrate backward in time to $t_{0}$. The gradients of the cost function with respect to the initial conditions $\mathbf{x}_{0}$ can thus be obtained. The correctness of the gradient calculation program module can be verified following:

$$
\Phi(\alpha)=\frac{J\left(\mathbf{x}_{0}+\alpha \Delta \mathbf{x}\right)-J\left(\mathbf{x}_{0}\right)}{\alpha \Delta \mathbf{x}^{\mathrm{T}} \nabla J\left(\mathbf{x}_{0}\right)}=1+O(\alpha),
$$
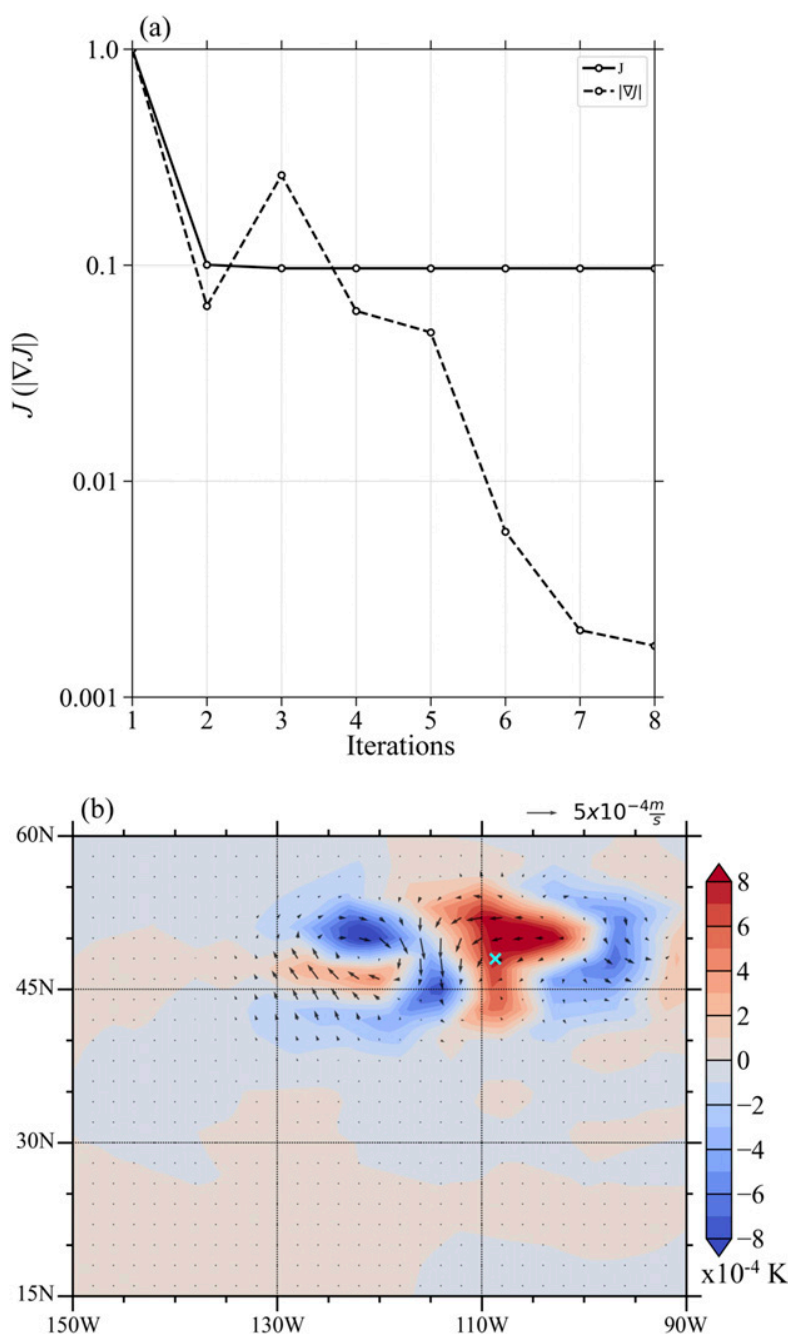

FIG. 5. (a) Evolutions of $J$ (solid curve) and the norm of $\nabla J$ (dashed curve) as a function of iteration in the experiment of assimilating a single observation. Both $J$ and $|\nabla J|$ have been normalized by their initial values. (b) Analysis increments of potential temperatures (shaded; K) and of wind vectors after assimilating the observation located at the cyan cross.

where $\Delta \mathbf{x}$ is a perturbation vector with the same dimension as $\mathbf{x}_{0}$, and $\alpha$ is a scaling factor. As the scaling factor $\alpha$ becomes smaller, the value of $\Phi(\alpha)$ is expected to approach unity linearly. Figure 2 shows the results of Eq. (6) for the MPAS-A 4DVar with a 6-h assimilation window, where the $x$ and $y$ axes are logarithmic. The calculations are configured for the quasiuniform-resolution (UR) and variable-resolution (VR) meshes shown in Fig. 1 following experiment A, described in more detail in section 3. The $|\Phi(a)-1|$ from the experiments for both UR (solid) and VR (dashed) meshes linearly decrease to zero as $\alpha$ gets smaller and smaller. When $\alpha$ becomes smaller than $10^{-8}$, machine round-off errors start to affect the results of Eq. (6), causing the linear relationship to disappear. Once the correctness of the gradient calculation is verified, both the modules of obtaining the cost functions and the gradients 
of the cost functions may be passed to a minimization algorithm to solve for analyses. Here, the limited-memory BroydenFletcher-Goldfarb-Shanno algorithm (L-BFGS) is used (Zhu et al. 1997). The practical implementation procedures include the following:

(i) providing a first-guess field $\mathbf{x}_{b}$;

(ii) computing the cost function $J$ and its gradient $\nabla_{\mathbf{x} 0} J$;

(iii) passing $J$ and $\nabla_{\mathbf{x} 0} J$ to a minimization algorithm of choice that will compute an updated $\mathbf{x}_{0}$ following the gradient of the cost function;

(iv) repeating steps ii and iii until a satisfactory convergence is achieved.

\section{4D-Var experimental design}

A common approach to estimate the background error (BE) covariance is the National Meteorological Center method, which essentially computes the differences between a pair of forecasts initialized at different times but both valid at the same time (Parrish and Derber 1992). In the case of MPAS-A 4D-Var, BE can be expressed as

$$
\mathbf{B}=\overline{\left(\mathbf{x}_{48}-\mathbf{x}_{24}\right)\left(\mathbf{x}_{48}-\mathbf{x}_{24}\right)^{\mathrm{T}}},
$$

where $\mathbf{x}_{48}$ and $\mathbf{x}_{24}$ are 48 - and 24 -h forecasts valid at the same time, respectively. The overbar represents averaging over time and space. In this study, the forecasts of 1 to 31 January 2020 are included in the calculation following Eq. (7). All off-diagonal elements of the full BE covariance matrix are assumed to be zero, implying that no spatial or multivariate correlations will be introduced by the background term in Eq. (4). For the purpose of better convergence when solving for the analysis, a preconditioning matrix $\mathbf{L}$, defined as $\mathbf{L} \mathbf{L}^{\mathrm{T}}=\mathbf{B}$, is given and multiplied by the state variables $\mathbf{x}$ to form a new variable $\mathbf{w}=\mathbf{L}^{-1} \mathbf{x}$. During the implementation of MPAS-A 4D-Var, w and its corresponding gradient will be handed over to the minimization algorithm to search for the minimum value of the cost function $J$. Here, since the BE covariance matrix B is diagonal, the elements in $\mathbf{L}$ are simply the square root of those in $\mathbf{B}$.

The first guess and observations are generated from the ECMWF's ERA5 reanalysis (Hoffmann et al. 2019). The ERA5 datasets at 0900 and 1200 UTC 1 February 2020 are first interpolated onto a global mesh with a uniform $480-\mathrm{km}$ resolution (Fig. 1a). The mesh consists of 2562 grid cells horizontally over the global domain, upon which interpolated atmospheric profiles have 55 vertical levels. The forecasts valid at 0000 UTC 2 February 2020 made by the nonlinear MPAS-A with ERA5 at 0900 UTC 1 February as initial conditions will serve as the first guess in the 4D-Var experiments. Forecasts with ERA5 at 1200 UTC 1 February as initial conditions and valid at 0000 and 0600 UTC 2 February will serve as the "truth" to be referenced by the analysis and "observations." No additional noise is added to the referenced "truth" or observations. Since the pseudo-observations are the model prognostic variables themselves, the observation operator $H_{r}$ is simply an identity
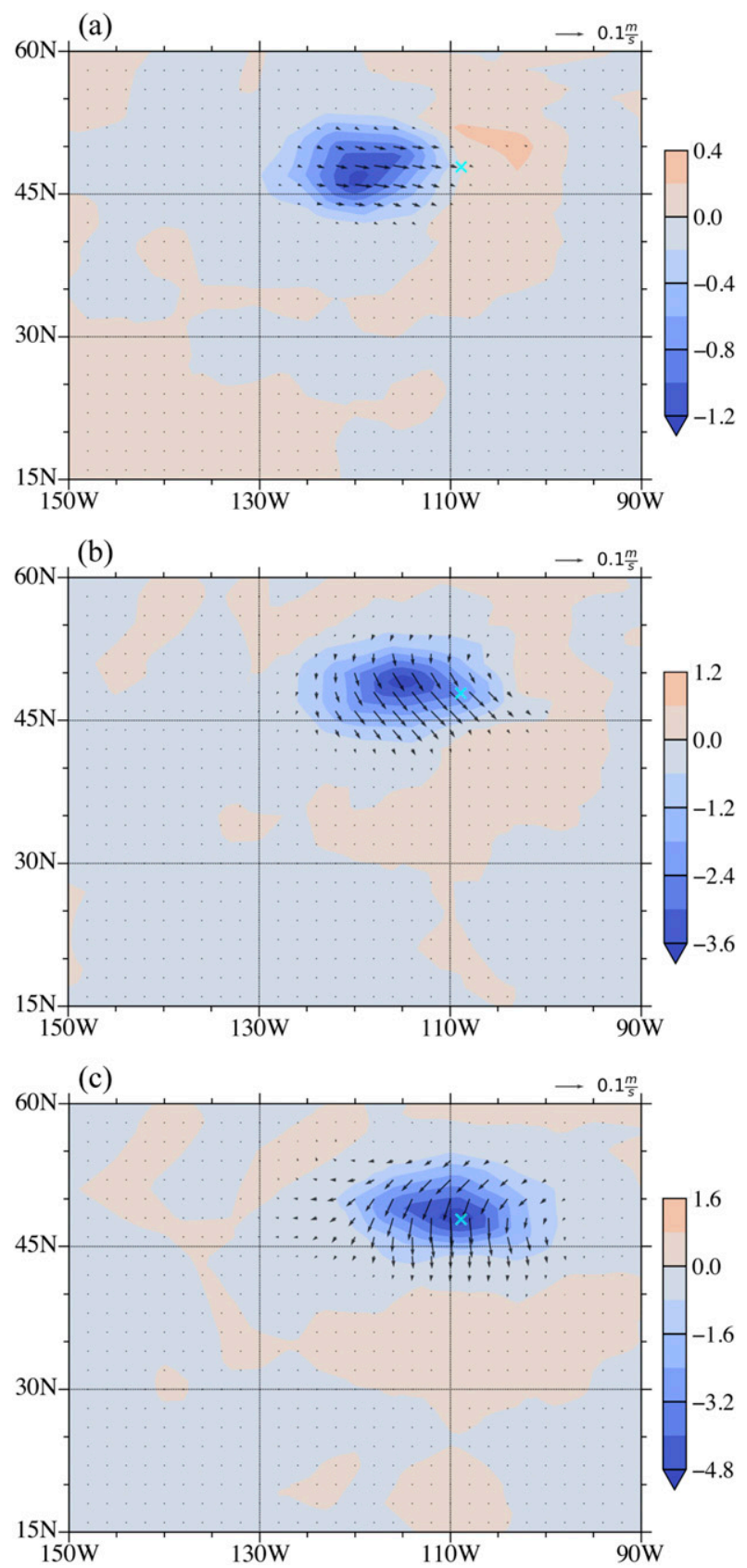

FIG. 6. Evolution of the analysis increments shown in Fig. 5b after (a) 2, (b) 4, and (c) $6 \mathrm{~h}$ of nonlinear forecasts. The location of the observation is marked by the cyan cross.

matrix $\mathbf{I}$ in this case. The observation errors $\mathbf{O}$ are given the same values as B estimated by Eq. (7). Similarly, for the experiments with a VR mesh consisting of 10242 grid cells (Fig. 1b) with resolutions ranging from 100 to $400 \mathrm{~km}$, the first guess, referenced "truth," and "observations" at 0000 UTC 11 February 2020 are generated with the ERA5 at 0900 and 1200 UTC 1 February. Figure 3 shows the time flow describing the experimental configurations, where the assimilation window is $6 \mathrm{~h}$. The "observations" take place at the end of the 

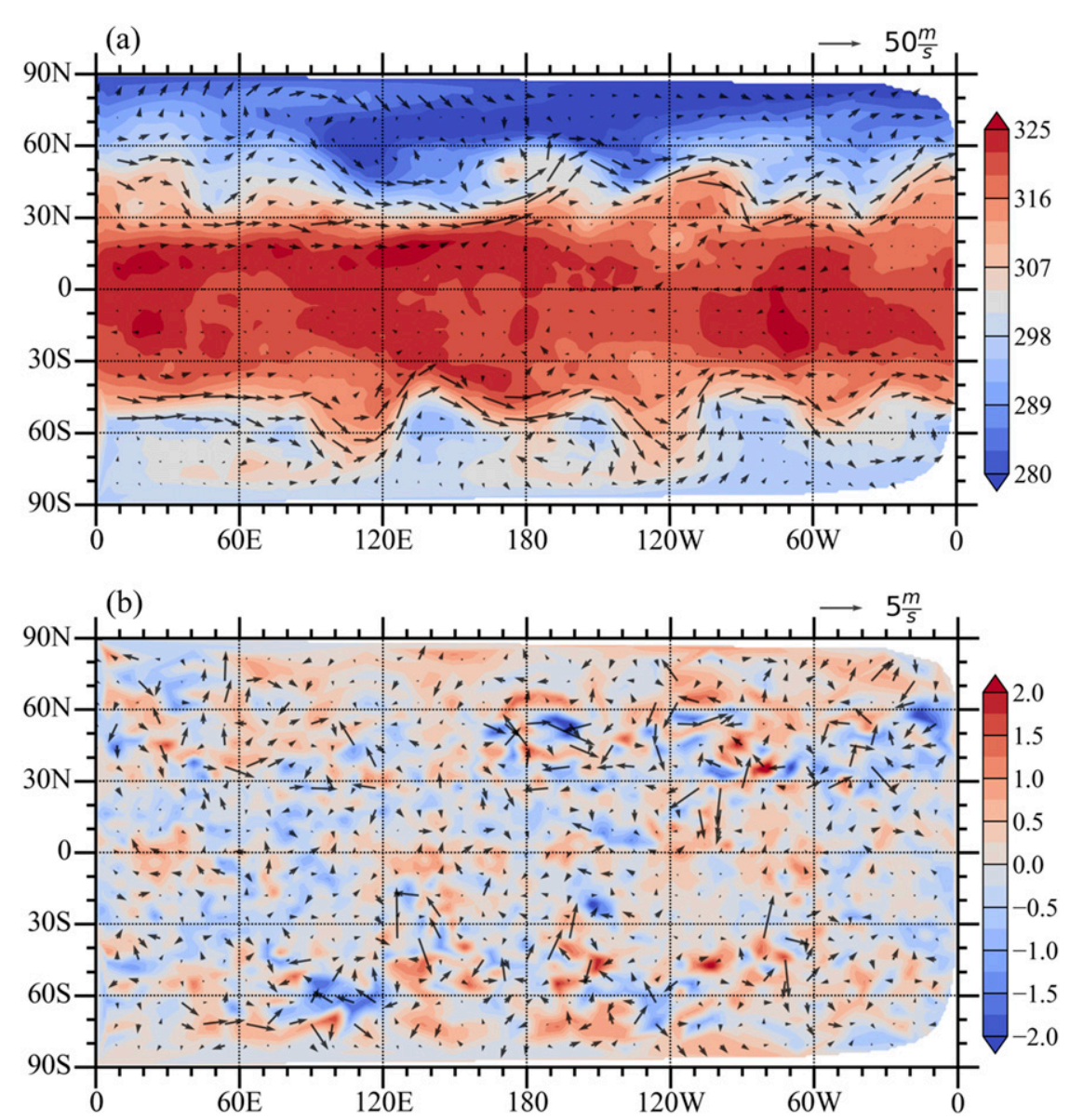

FIG. 7. (a) Spatial distributions of potential temperatures (shaded; K) and wind vectors at 0000 UTC 2 Feb 2020 from the forecast with 1200 UTC 1 Feb 2020 as initial conditions, i.e., the reference. (b) Differences between the first guess and the reference shown in (a).

assimilation window. Three separate experiments are then conducted with the above-generated first guess and observations that include

1) single-point observation assimilation, where $\theta$ at one location is observed and assimilated;

2) full-state-vector-observation assimilation to reversely infer the initial state, where "observations" of all five model prognostic variables over the entire global domain are assimilated;

3) mass-observation assimilation for wind-field reconstructions, where only values of $\theta$ over the global domain or a region are observed and assimilated.

\section{Analysis results}

\section{a. Experiments with the UR mesh}

\section{1) SINGLE-POINT OBSERVATION}

As described in section 3, a single-point observation valid at 0600 UTC 2 February 2020 is assimilated to solve for the analysis at 0000 UTC 2 February. Figure 4 shows the spatial distributions of $\theta$ and wind vectors at $500 \mathrm{hPa}$ in the first guess at 0000 UTC and the observations at 0600 UTC. During the 6-h assimilation window, the trough at the latitude band of about $45^{\circ} \mathrm{N}$ slowly propagated eastward. The cyan cross in Fig. $4 \mathrm{~b}$ marks the one observation point's location, where only the value of $\theta$ will be assimilated in this experiment. Figure 5a shows the evolution of the cost function and the norm of the gradient. The value of the cost function decreased by more than one order of magnitude, and the gradient's norm decreased by nearly three orders of magnitude, suggesting an effective convergence. Figure $5 \mathrm{~b}$ illustrates the increments in the resulting analysis. Even though only the $\theta$ at a single location is assimilated, in addition to increments in $\theta$, anticyclonicpatterned adjustments to the wind field can also be found upstream from the location of the observation. Flow-dependent features are seen in both fields because the gradient of the cost function is calculated by the MPAS-A AD model following the trajectory generated by the nonlinear MPAS-A model (Gustafsson 2007). Figure 6 shows the differences between the nonlinear forecasts taking the first guess and analysis as initial conditions, demonstrating how the analysis increment evolved 


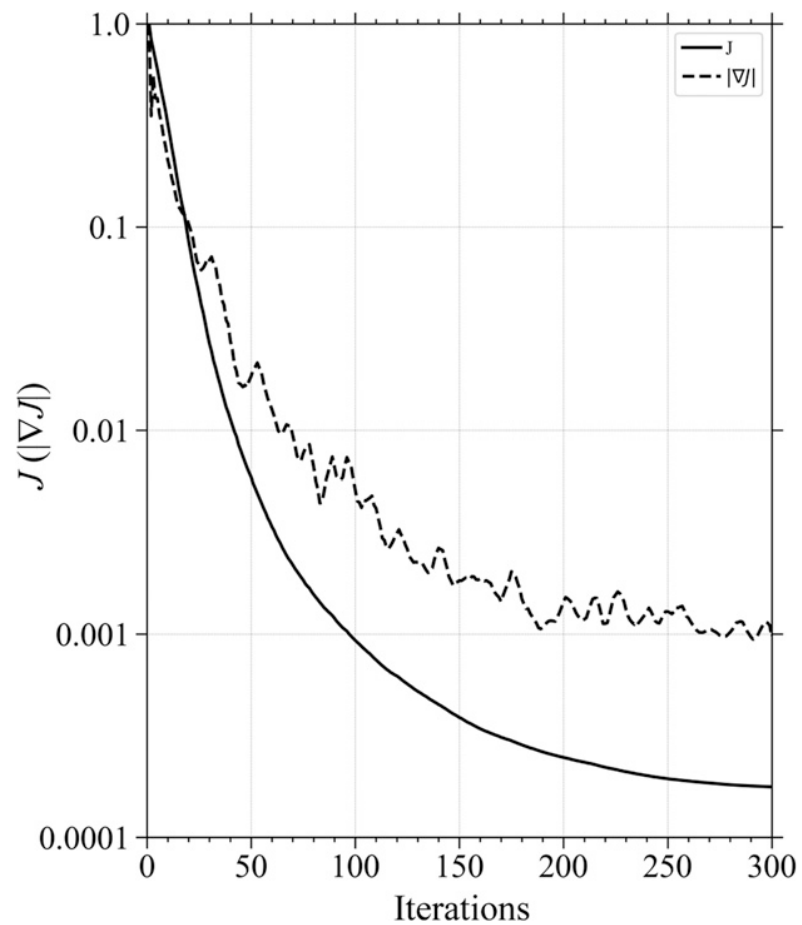

FIG. 8. Variations in $J$ (solid curve) and the norm of $\nabla J$ (dashed curve) as a function of iteration in the experiment assimilating fullvector observations. Both $J$ and $|\nabla J|$ have been normalized by their initial values.

within the assimilation window, specifically at 2, 4, and $6 \mathrm{~h}$. Both $\theta$ and wind vector fields gradually propagated toward the observation location, settling down around the observation at the end of the assimilation window. As Bannister (2008) described, in 4D-Var, the BE covariance is implicitly propagated following the atmospheric flow to the time of observations within the assimilation window. However, the $\mathbf{B}$ matrix reverts back to a static one at the start of the assimilation window.

\section{2) INFERENCE OF THE INITIAL STATE}

In this experiment, the "observations" consist of the complete atmospheric state, i.e., all five prognostic model variables $u, w, \rho, \theta$, and $q_{v}, 6 \mathrm{~h}$ after the time of analysis. The availability of full-vector observations in this experiment is a highly idealized and rare scenario where Eq. (4) becomes an adequately determined problem even without the term $J_{b}$. Thus, in this specific experiment only, the background terms in Eqs. (4) and (5) are omitted (Žagar et al. 2004), reducing these equations to

$$
\begin{gathered}
J=J_{o}=\frac{1}{2} \sum_{r=0}^{N}\left[H_{r}\left(\mathbf{x}_{r}\right)-\mathbf{y}_{r}\right]^{\mathrm{T}} \mathbf{O}_{r}^{-1}\left[H_{r}\left(\mathbf{x}_{r}\right)-\mathbf{y}_{r}\right], \\
\nabla J=\sum_{r=0}^{N} \mathbf{M}_{r}^{\mathrm{T}} \mathbf{H}_{r}^{\mathrm{T}} \mathbf{O}_{r}^{-1}\left[H_{r}\left(\mathbf{x}_{r}\right)-\mathbf{y}_{r}\right] .
\end{gathered}
$$

The purpose of such a setup is to try to reconstruct the atmospheric state at the analysis time based on future
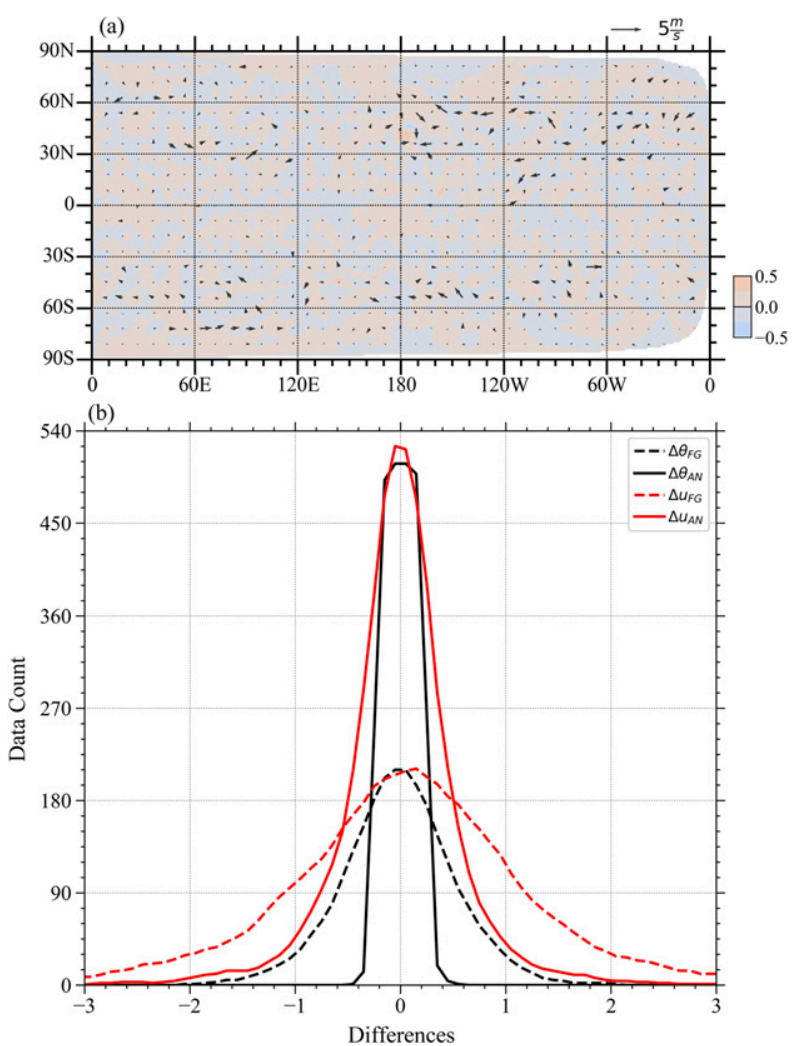

FIG. 9. (a) Differences between the analysis after assimilating the full-vector observations and the reference shown in Fig. 6a. (b) Histograms of the differences in potential temperature (black curves) and wind (red curves) before (dashed) and after (solid) assimilating the full-vector observations.

observations. In this study, the complete atmospheric state at the time of observation is generated with the same MPAS-A nonlinear forecast model as in the 4D-Var DA system. The solved analysis and the known referenced "truth" should thus be similar. Figure 7a shows the spatial distributions of $\theta$ and wind vectors over the global domain in the first guess. Differences between the first guess and the known reference are given in Fig. 7b. Although random in general, the largest magnitudes of the differences in both mass and wind fields tend to appear in mid and high-latitude regions, where most planetary waves are located.

Figure 8 shows variations in the cost function and the norm of the gradient in the minimization process. The convergence is not as rapid as in the case of the single-point observation. The "observations" include all five model variables over the entire globe, so the gradients of the cost function are bound to be much more complicated than in the case of one observation. Overall, after 300 iterations, the cost function decreased by more than three orders of magnitude, and the norm of the gradient decreased by about three orders of magnitude, comparable to results reported by Thepaut and Courtier (1991) using a global primitive and adiabatic equation model. Figure $9 \mathrm{a}$ shows differences like those in Fig. $7 b$, except that the 


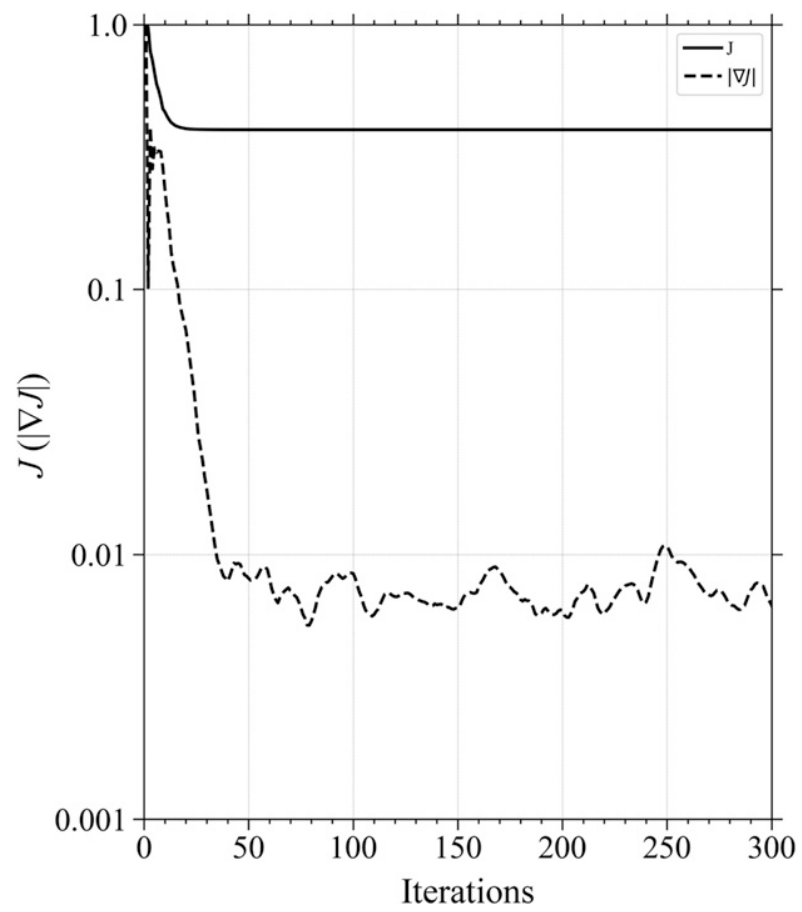

FIG. 10. Variations in $J$ (solid curve) and the norm of $\nabla J$ (dashed curve) as a function of iteration in the experiment assimilating mass field observations. Both $J$ and $|\nabla J|$ have been normalized by their initial values.

observations $6 \mathrm{~h}$ after the analysis time are assimilated. The magnitudes of the differences in both mass and wind fields are substantially reduced. Statistically, the histogram of the differences shows that the majority of the differences in $\theta$ are within the range of $[-0.4,0.4 \mathrm{~K}]$. The wind field also shrinks noticeably toward zero, indicating that both fields are well reconstructed (Fig. 9b).

This experiment's configuration is highly ideal because observations rarely cover all model variables and the entire global domain. At the same time, the assimilation of fullvector observations $6 \mathrm{~h}$ after the analysis time under such settings is exceedingly difficult, as evidenced by the convergence rate in the minimization process. However, the final convergence and a close reconstruction of the analysis with respect to the referenced "truth" validates the numerical feasibility of the global 4D-Var DA system proposed in this study.

\section{3) WIND RECONSTRUCTION WITH THE MASS FIELD}

Unlike the second experiment, only the $\theta$ field over the global domain $6 \mathrm{~h}$ after the analysis time is assimilated. Temperature is among the most commonly observed variables in realistic situations (Tian and Zou 2016, 2018; Zou and Tian 2018). Figure 10 shows the convergence rate of the minimization. After 300 iterations, the norm of the gradient of the cost function decreased by more than two orders of magnitude. Figure 11a shows the spatial distributions of the differences between the resulting analysis and the reference. Compared
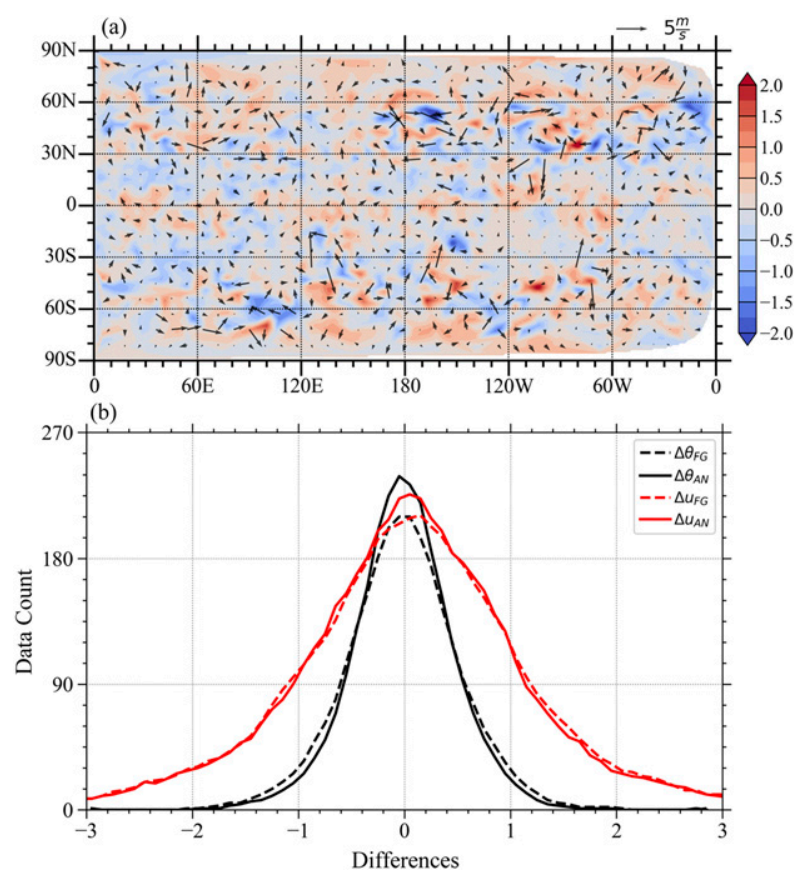

FIG. 11. (a) Differences between the analysis after assimilating the potential temperatures and the reference shown in Fig. 6a. (b) Histograms of differences in potential temperature (black curves) and wind vectors (red curves) before (dashed) and after (solid) assimilating potential temperatures.

with Fig. 7b, differences in $\theta$ are slightly smaller in magnitude, and differences in wind vectors appear negligible. Figure $11 \mathrm{~b}$ shows the histogram of the differences before and after assimilation. The differences in both variables shrink toward zero to some extent, more in the case of $\theta$ than in wind. Figure 12 shows the analysis increments of $\theta$ and wind compared to the first guess (Fig. 12a) and the mean and standard deviations of the analysis increments of wind with respect to latitude in $5^{\circ}$ bins (Fig. 12b). Both the means and standard deviations of the increments in the tropics are smaller than those at higher latitudes. These statistics agree with the geostrophic adjustment theory that adjustments of wind by assimilating only mass observations should be slower close to the equator (Holton 1973; Žagar et al. 2004). Because only $\theta$ are "observed," the wind field is still reconstructed to a statistically meaningful extent, one of the highly sought-after advantages of 4D-Var, namely, that dynamical consistency following the atmospheric governing equations can be maintained in the solved analysis.

\section{b. Experiments with the VR mesh}

Experiments A, B, and C with some modifications are conducted using a smoothly VR mesh. For experiment $\mathrm{A}, \theta$ values over two individual points at $500 \mathrm{hPa}$ are assimilated, one inside a finely resolved area and the other inside a coarsely resolved area (Fig. 13b with the Voronoi grid distributions overlaid). Figure 13a shows the evolution of the cost function and the norm of its gradient with respect to iteration. The 


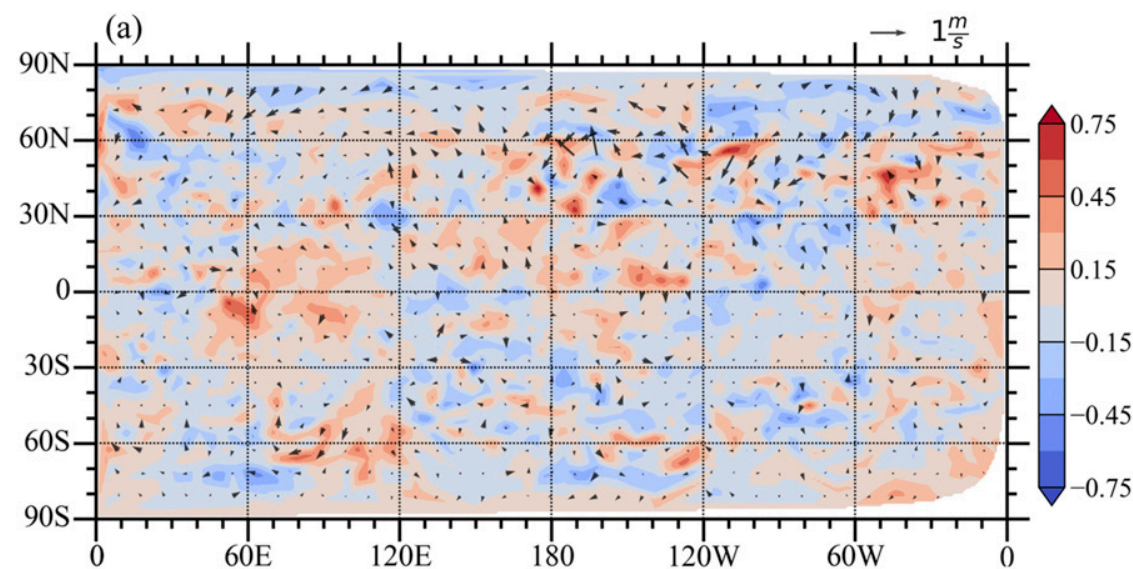

(b)

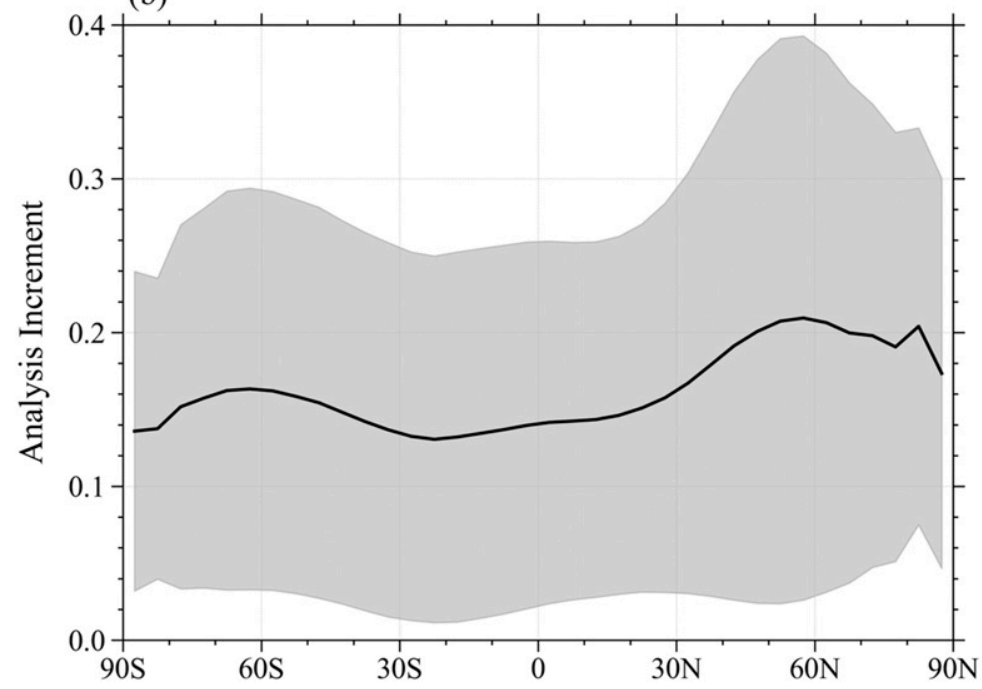

FIG. 12. (a) Analysis increments of potential temperature (shaded) and wind vectors (arrows) after assimilating only potential temperature. (b) Means (black curve) and standard deviations (shaded area) within each $5^{\circ}$ latitude bin of the analysis increments of winds shown in (a).

gradient of the cost function decreased by more than two orders of magnitude after 19 iterations. The locations of the observations are marked by cyan crosses in Fig. 13a. Although only $\theta$ values at these two locations are assimilated, adjustments to the initial conditions are manifested in both the $\theta$ and wind fields upstream of the observation locations. With the VR mesh, flow dependency in the adjustments following atmospheric dynamics can be obtained regardless of the observation locations and the underlying resolutions. Figure 14 shows the evolution of the analysis increments in the nonlinear forecasts after 2, 4, and $6 \mathrm{~h}$. The $\theta$ and wind vectors in both patterns gradually moved eastward following the nonlinear trajectory, stopping at the observation locations.

Figure 15 shows the results of assimilating full-vector observations $6 \mathrm{~h}$ after the analysis time using the VR mesh. As demonstrated in Fig. 15a, after 300 iterations, the cost function is reduced by about three orders of magnitude and its gradient by about two orders of magnitude. Similar to Fig. 9b, the histogram of the differences with respect to the referenced truth before and after DA is shown in Fig. 15b. The distribution of the differences in both $\theta$ and wind under initial conditions shrink considerably toward zero when compared with the reference after DA, indicating an effective inference of the complete initial state of the atmosphere from observations available $6 \mathrm{~h}$ later.

Different from experiment $\mathrm{C}$ using the UR mesh, here with the VR mesh, the $\theta$ values of only one region are assimilated (Fig. 16b). Figure 16 shows the resulting cost functions, norms of their gradients, and analysis increments. Similar to that shown in Fig. 10, the norms of the gradients in Fig. 16a decreased by more than two orders of magnitude but only after 35 iterations. The region selected here (outlined by the oval in Fig. 16b) covers an area with grid cells that are both at fine resolution and at resolutions that gradually coarsen. This was done to demonstrate coherent 

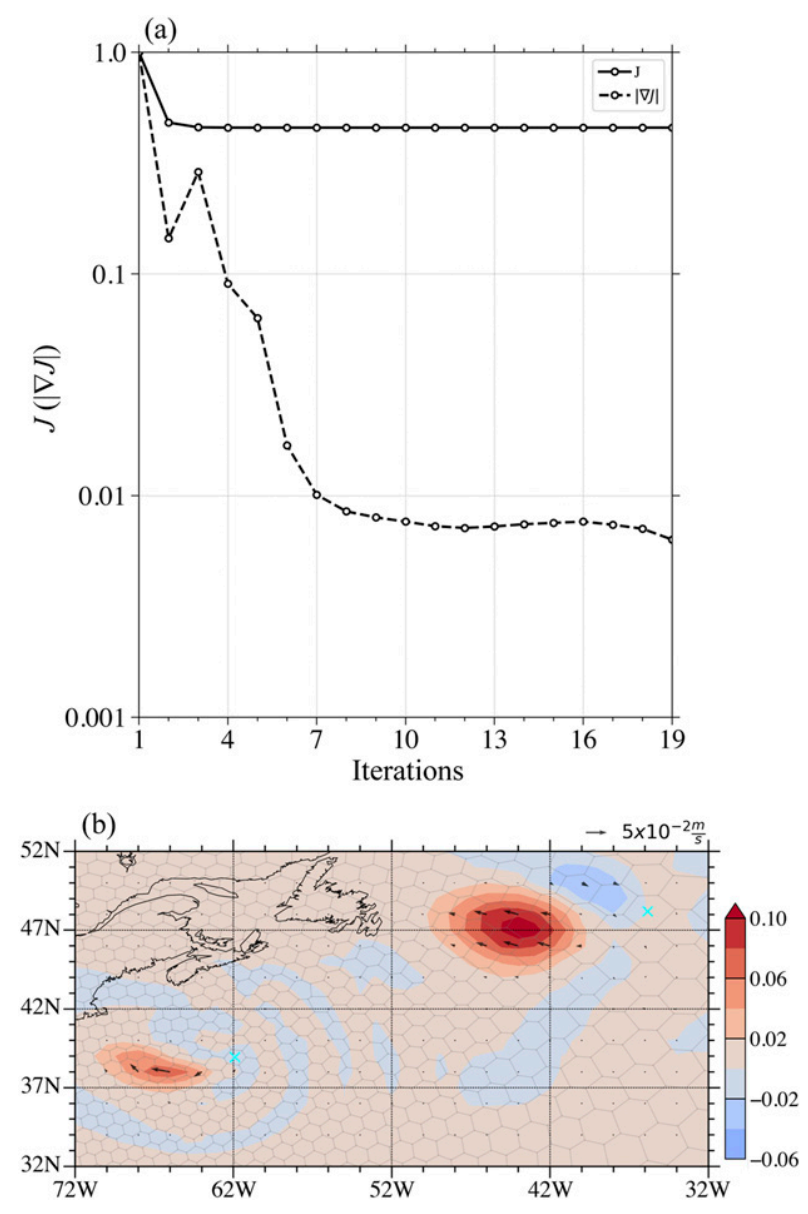

FIG. 13. (a) Evolutions of $J$ (solid curve) and the norm of $\nabla J$ (dashed curve) as a function of iteration in the experiment of assimilating two observations using the VR mesh. Both $J$ and $|\nabla J|$ have been normalized by their initial values. (b) Analysis increments of potential temperature (shaded) and wind vectors after assimilating the observations marked by the two cyan crosses. The actual Voronoi grid cells are overlaid in gray.

analysis increment features in MPAS-A 4D-Var. Areas under initial conditions that are sensitive to the selected region $6 \mathrm{~h}$ after are found both inside and outside the oval. The analysis increment patterns also span various resolutions, indicating that the simulations within the oval are subject to influence from signals resolved at different scales.

\section{Summary and conclusions}

This study briefly documents the development of a global 4D-Var DA system with the MPAS-A model. The MPAS-A nonlinear forecast model was combined with the MPAS-A AD model described by Tian and Zou (2020) to formulate a continuous 4D-Var DA framework. A scalar cost function was defined, measuring the distances of the analysis vector to the background state vector and to the observations potentially distributed at different times. The gradient of the cost function with respect to the analysis was then calculated using the MPAS-A AD model. A necessary correctness procedure verifying the gradient calculations was implemented to ensure the accuracy of the gradients so that minimization algorithms could search in the right direction. The limitedmemory BFGS was adopted for finding the minima of the cost functions in the experiments carried out in this study.

Three idealized experiments were conducted to validate and demonstrate the numerical feasibility of the MPAS-A 4D-Var DA system. The first guess, the "observations" $6 \mathrm{~h}$ after the analysis time, and the referenced "truth" valid for the analysis time were generated using the MPAS-A nonlinear forecast model taking ERA5 from the ECMWF as initial conditions. In the first experiment, the observation consisted of only $\theta$ at a single point. The analysis increment demonstrates a flow-dependent feature in both the wind vectors and $\theta$ values even though only one $\theta$ was "observed" and assimilated. In the second experiment, observations spanned the full analysis vector, i.e., all model prognostic variables over the entire global domain. In this experiment, perfect observations were assumed, implying that no background error covariances were involved. By assimilating fullvector observations $6 \mathrm{~h}$ after the analysis time, the initial state used to generate the observations was expected to be reconstructed by the $4 \mathrm{D}-$ Var DA system. After minimizations, the cost function (norm of the gradient) decreased by more than (about) three orders of magnitude. The differences between the analysis and the reference in wind vectors and $\theta$ were significantly smaller than those between the first guess and the reference. This experiment, although idealized, suggests that the 4D-Var DA system is numerically capable of solving for the analysis in challenging situations. The third experiment shows that the assimilation of mass field observations can also contribute to the reconstruction of the wind field, retaining dynamical consistency in the analysis. Results show that both the wind and $\theta$ in the analysis statistically agree more with the reference than the first guess. A similar set of experiments was conducted using a smoothly VR mesh. Flow-dependent features corresponding to individual observations in both fine- and coarse-resolution grids were found in the initial conditions. In the case of assimilating full-vector observations, initial conditions agreeing considerably better with the reference could be inferred from observations available $6 \mathrm{~h}$ after. In the third experiment, only $\theta$ values within a selected region were assimilated to demonstrate the coherence as well as the flow dependency in the analysis increment in regional DA applications.

The 4D-Var DA system proposed in this study is structured with a Python driver layer that can conveniently manage components such as input/output, control of simulation time flows, and matrix manipulations. A FORTRAN modeling layer is responsible for the heavy-duty numerical calculations. Such a structure retains a fast computational efficiency while bringing in the advantageous features of Python. Any future additions to this DA system, such as more efficient optimization modules, observation operators, radiative transfer models, and physical parameterizations, can be readily incorporated into the current structure. 
(a)

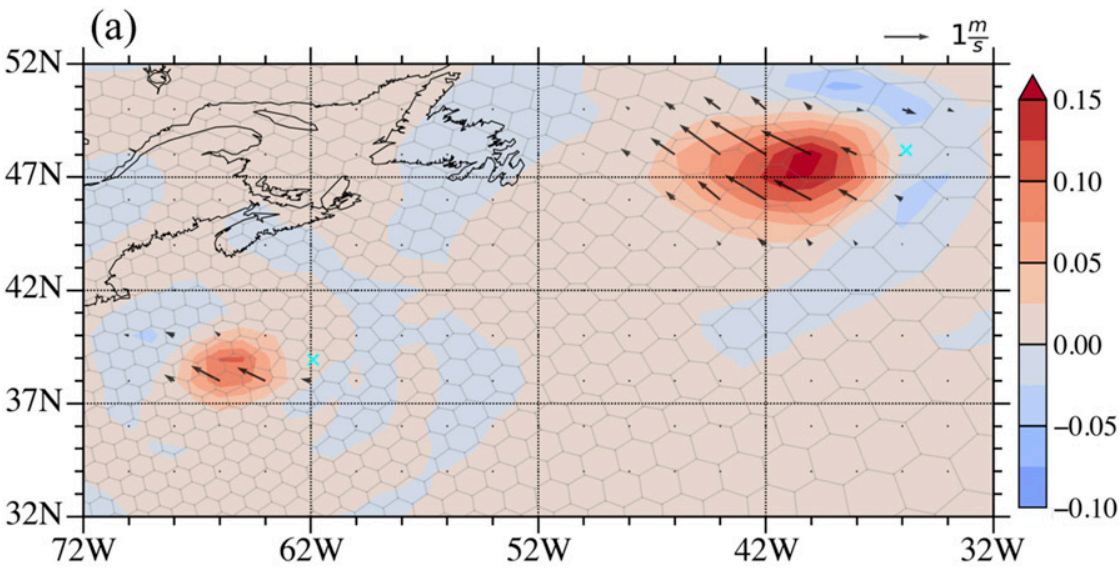

(b)

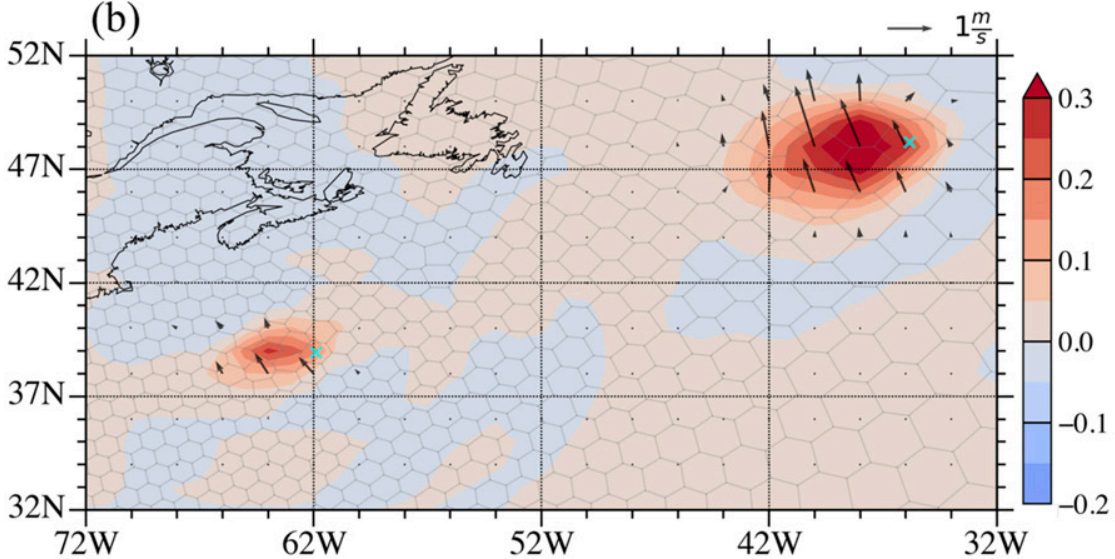

(c)

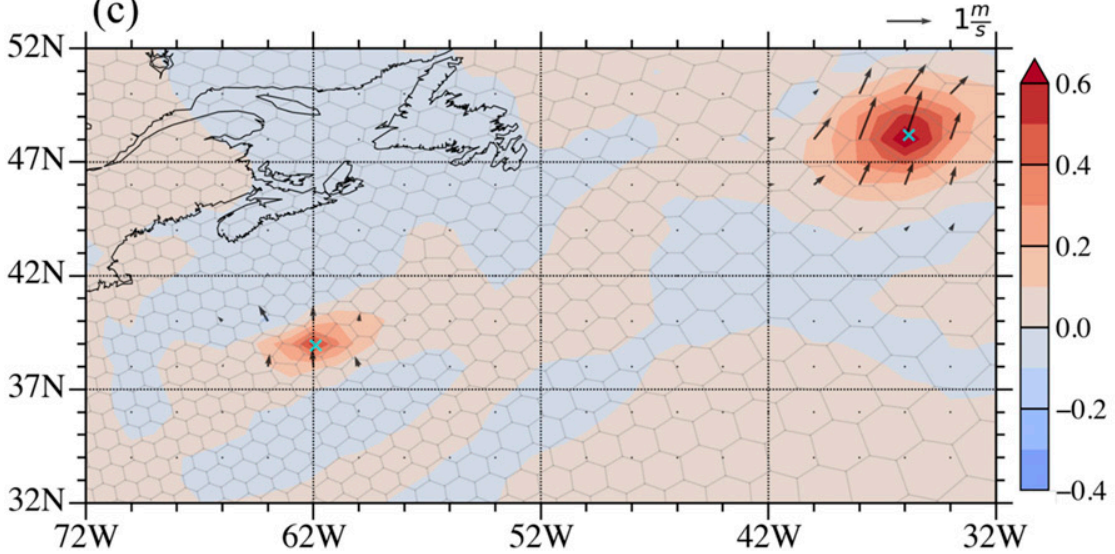

FIG. 14. Evolution of the analysis increments of potential temperatures (shaded) and wind vectors shown in Fig. 13b after (a) 2, (b) 4, and (c) $6 \mathrm{~h}$ of nonlinear forecasts. The locations of the observations are marked by the two cyan crosses. 
(a)

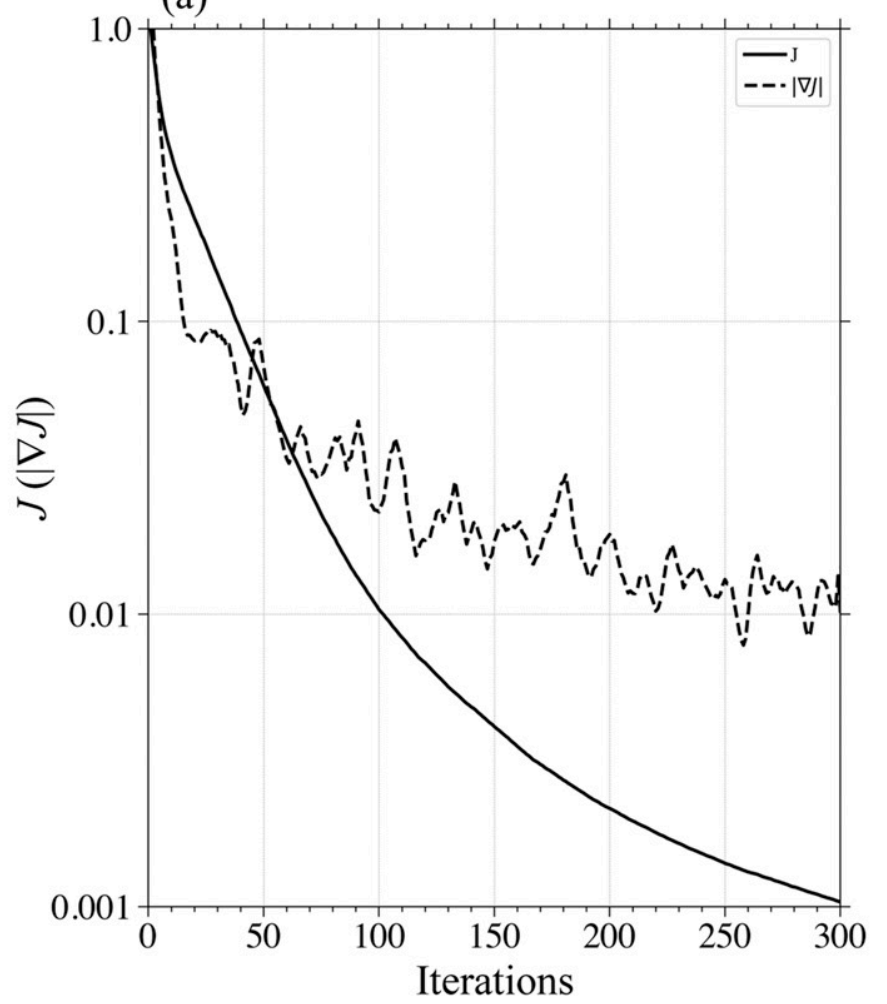

(b)

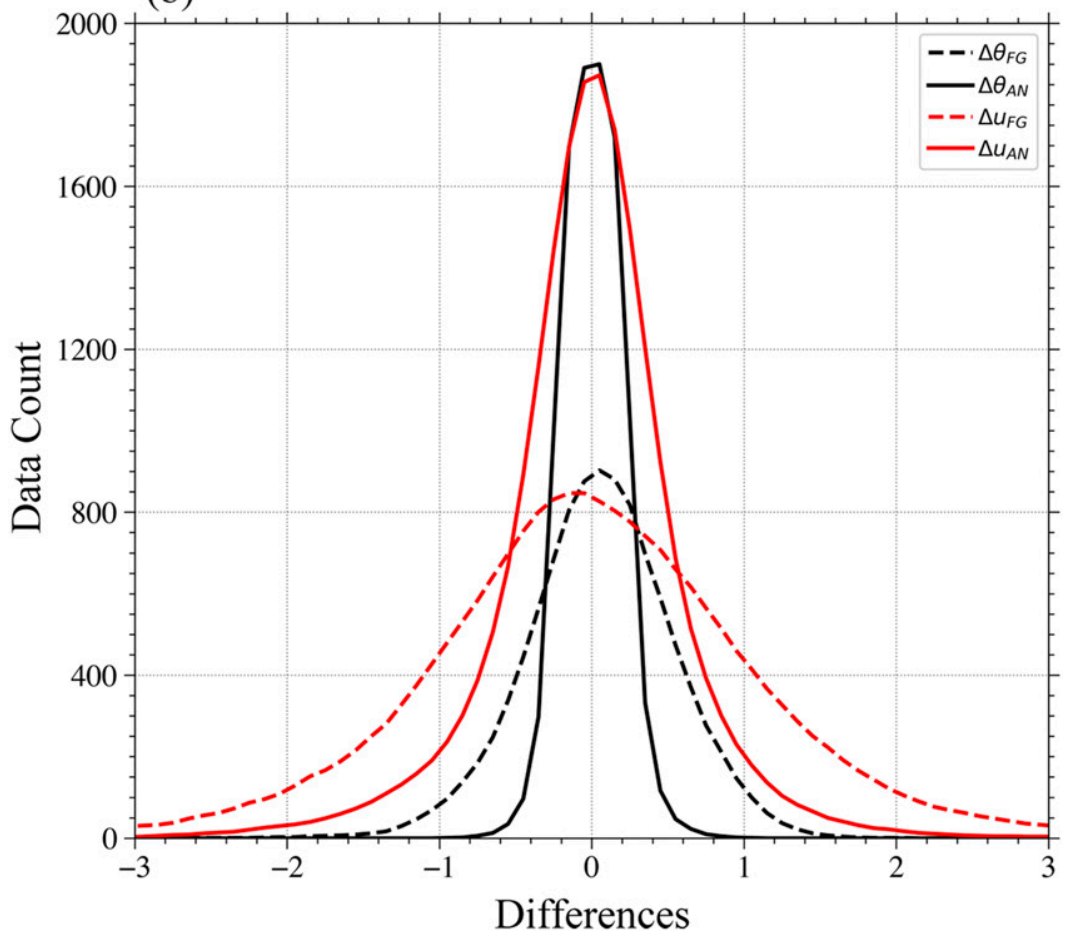

FIG. 15. (a) Variations in $J$ (solid curve) and the norm of $\nabla J$ (dashed curve) as a function of iteration in the experiment of assimilating full-vector observations using the VR mesh. Both $J$ and $|\nabla J|$ have been normalized by their initial values. (b) Histograms of the differences in potential temperature (black curves) and wind (red curves) before (dashed) and after (solid) assimilating the full-vector observations. 
(a)

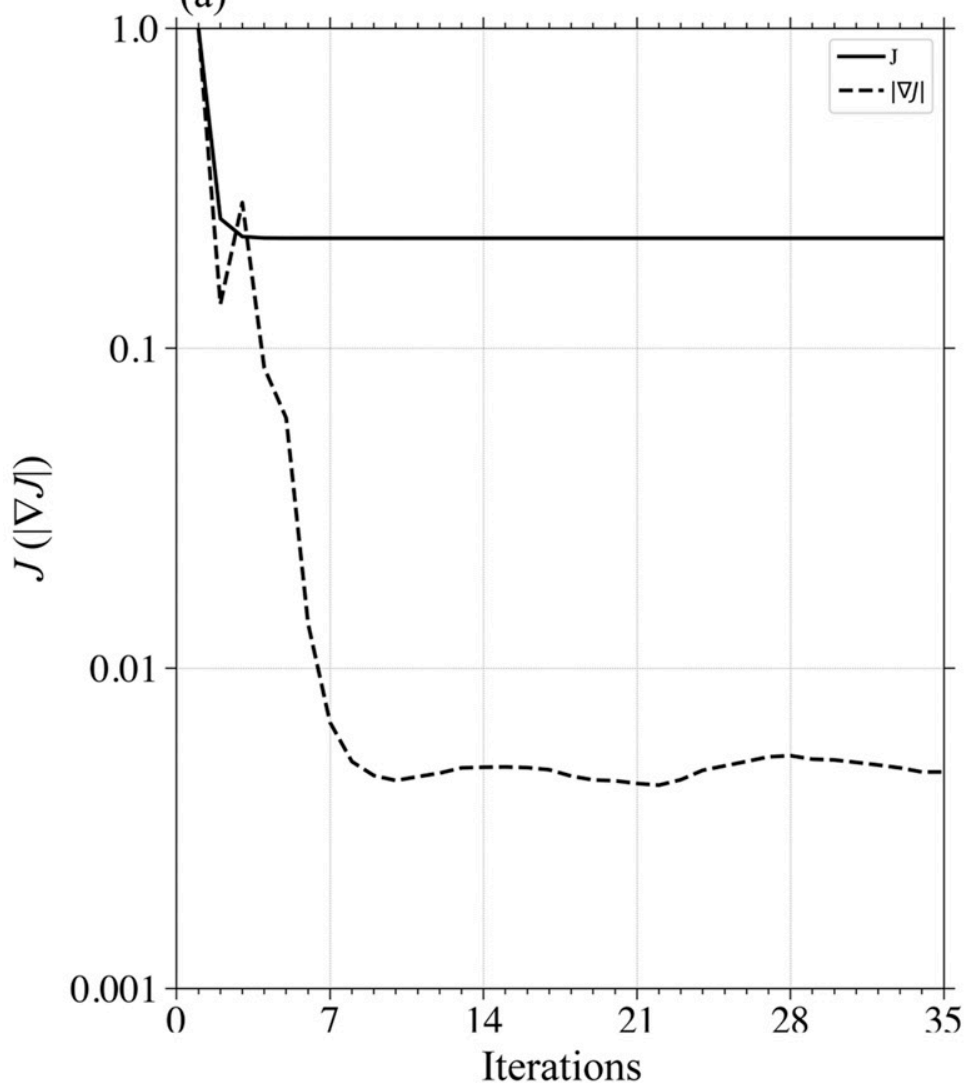

(b)

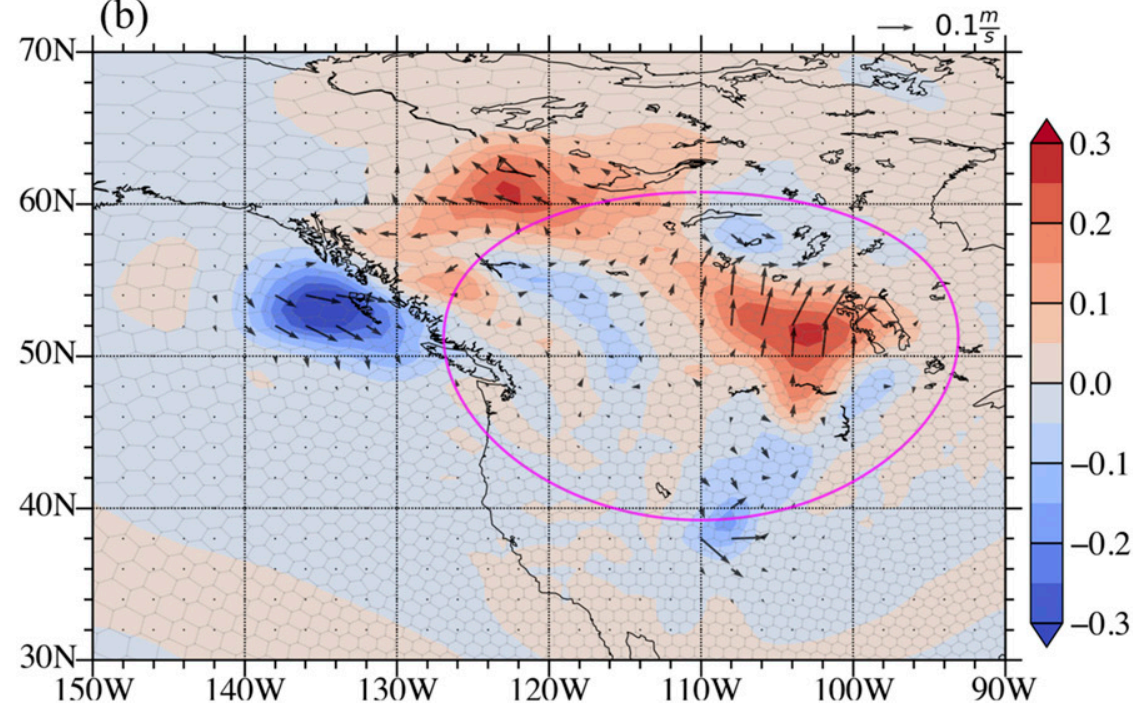

FIG. 16. (a) Variations in $J$ (solid curve) and the norm of $\nabla J$ (dashed curve) as a function of iteration in the experiment of assimilating potential temperatures in the region outlined by the purple oval in (b). Both $J$ and $|\nabla J|$ have been normalized by their initial values. (b) Analysis increments of potential temperatures (shaded) and wind vectors. 
Acknowledgments. The authors thank NCAR for releasing the source code of MPAS-Atmosphere at https://mpasdev.github.io/. Thanks also go to Michael Duda and Darren Engwirda for their help in preparing the variable-resolution mesh. The authors appreciate the review comments from the editor and reviewers that helped to improve this study. The second author is supported by the National Key R\&D Program of China (Grant 2018YFC1507004). Data for producing the plots in this manuscript are available at https:// www.xiaoxutian.com/products/.

\section{REFERENCES}

Bannister, R. N., 2008: A review of forecast error covariance statistics in atmospheric variational data assimilation. I: Characteristics and measurements of forecast error covariances. Quart. J. Roy. Meteor. Soc., 134, 1951-1970, https:// doi.org/10.1002/qj.339.

Courtier, P., J. Thépaut, and A. Hollingsworth, 1994: A strategy for operational implementation of 4D-Var, using an incremental approach. Quart. J. Roy. Meteor. Soc., 120, 1367-1387, https:// doi.org/10.1002/qj.49712051912.

Gauthier, P., and J.-N. Thepaut, 2001: Impact of the digital filter as a weak constraint in the preoperational 4DVar assimilation system of Météo-France. Mon. Wea. Rev., 129, 2089-2102, https://doi.org/10.1175/1520-0493(2001)129<2089:IOTDFA> 2.0.CO;2.

Gustafsson, N., 2006: Status and performance of HIRLAM 4D-Var. HIRLAM Newsl., 51, 8-16.

— 2007: Discussion on '4D-Var or EnKF?'. Tellus, 59A, 774777, https://doi.org/10.1111/j.1600-0870.2007.00262.x.

_- X.-Y. Huang, X. Yang, K. Mogensen, M. Lindskog, O. Vignes, T. Wilhelmsson, and S. Thorsteinsson, 2012: Four-dimensional variational data assimilation for a limited area model. Tellus, 64A, 14985, https://doi.org/10.3402/tellusa.v64i0.14985.

Hagos, S., R. Leung, S. A. Rauscher, and T. Ringler, 2013: Error characteristics of two grid refinement approaches in aquaplanet simulations: MPAS-A and WRF. Mon. Wea. Rev., 141, 3022-3036, https://doi.org/10.1175/MWR-D-12-00338.1.

Hoffmann, L., and Coauthors, 2019: From ERA-Interim to ERA5: The considerable impact of ECMWF's nextgeneration reanalysis on Lagrangian transport simulations. Atmos. Chem. Phys., 19, 3097-3124, https://doi.org/10.5194/acp19-3097-2019.

Holton, J. R., 1973: An introduction to dynamic meteorology. Amer. J. Phys., 41, 752-754, https://doi.org/10.1119/1.1987371.

Huang, X.-Y., and Coauthors, 2009: Four-dimensional variational data assimilation for WRF: Formulation and preliminary results. Mon. Wea. Rev., 137, 299-314, https://doi.org/10.1175/ 2008MWR2577.1.

Le Dimet, F.-X., and O. Talagrand, 1986: Variational algorithms for analysis and assimilation of meteorological observations: Theoretical aspects. Tellus, 38A, 97-110, https://doi.org/10.3402/ tellusa.v38i2.11706.

Lewis, J. M., and J. C. Derber, 1985: The use of adjoint equations to solve a variational adjustment problem with advective constraints. Tellus, 37A, 309-322, https://doi.org/10.3402/tellusa.v37i4.11675.

Lin, J. W.-B., 2012: Why python is the next wave in earth sciences computing. Bull. Amer. Meteor. Soc., 93, 1823-1824, https:// doi.org/10.1175/BAMS-D-12-00148.1.

Lorenc, A. C., and F. Rawlins, 2005: Why does 4D-Var beat 3D-Var? Quart. J. Roy. Meteor. Soc., 131, 3247-3257, https:// doi.org/10.1256/qj.05.85.
Lorenz, E., 1975: Climatic predictability. The Physical Basis of Climate and Climate Modelling, B. Bolin et al., Eds., World Meteorological Organization, 132-136.

Michaelis, A. C., G. M. Lackmann, and W. A. Robinson, 2019: Evaluation of a unique approach to high-resolution climate modeling using the Model for Prediction Across Scales-Atmosphere (MPAS-A) version 5.1. Geosci. Model Dev., 12, 3725-3743, https://doi.org/10.5194/gmd12-3725-2019.

Navon, I. M., X. Zou, J. Derber, and J. Sela, 1992: Variational data assimilation with an adiabatic version of the NMC spectral model. Mon. Wea. Rev., 120,1433-1446, https://doi.org/10.1175/ 1520-0493(1992)120<1433:VDAWAA >2.0.CO;2.

Park, S.-H., W. C. Skamarock, J. B. Klemp, L. D. Fowler, and M. G. Duda, 2013: Evaluation of global atmospheric solvers using extensions of the Jablonowski and Williamson Baroclinic wave test case. Mon. Wea. Rev., 141, 3116-3129, https:// doi.org/10.1175/MWR-D-12-00096.1.

Parrish, D. F., and J. C. Derber, 1992: The National Meteorological Center's spectral statistical-interpolation analysis system. Mon. Wea. Rev., 120, 1747-1763, https://doi.org/10.1175/15200493(1992)120<1747:TNMCSS $>2.0$. CO;2.

Skamarock, W. C., J. B. Klemp, M. G. Duda, L. D. Fowler, S.-H. Park, and T. D. Ringler, 2012: A multiscale nonhydrostatic atmospheric model using centroidal Voronoi tesselations and C-grid staggering. Mon. Wea. Rev., 140, 3090-3105, https:// doi.org/10.1175/MWR-D-11-00215.1.

Thepaut, J., and P. Courtier, 1991: Four-dimensional variational data assimilation using the adjoint of a multilevel primitiveequation model. Quart. J. Roy. Meteor. Soc., 117, 1225-1254, https://doi.org/10.1002/qj.49711750206.

Tian, X., and X. Zou, 2016: ATMS-and AMSU-A-derived hurricane warm core structures using a modified retrieval algorithm. J. Geophys. Res. Atmos., 121, 12 630-12 646, https:// doi.org/10.1002/2016JD025042.

— , and — 2018: Capturing size and intensity changes of Hurricanes Irma and Maria (2017) from polar-orbiting satellite microwave radiometers. J. Atmos. Sci., 75, 2509-2522, https://doi.org/10.1175/JAS-D-17-0315.1.

— , and —, 2019: A comprehensive 4D-Var vortex initialization using a nonhydrostatic axisymmetric TC model with convection accounted for. Tellus, $\mathbf{7 1 A}, 1-15$, https://doi.org/ 10.1080/16000870.2019.1653138.

adjoint models of the MPAS-Atmosphere dynamic core and applications in adjoint relative sensitivity studies. Tellus, 72A, 1-17, https://doi.org/10.1080/16000870.2020. 1814602.

Žagar, N., N. Gustafsson, and E. Källén, 2004: Dynamical response of equatorial waves in four-dimensional variational data assimilation. Tellus, 56A, 29-46, https://doi.org/10.1111/j.16000870.2004.00036.x.

Zhang, L., and Coauthors, 2019: The operational global fourdimensional variational data assimilation system at the China meteorological administration. Quart. J. Roy. Meteor. Soc., 145, 1882-1896, https://doi.org/10.1002/ qj.3533.

Zhu, C., R. H. Byrd, P. Lu, and J. Nocedal, 1997: Algorithm 778: L-BFGS-B: Fortran subroutines for large-scale boundconstrained optimization. ACM Trans. Math. Software, 23, 550-560, https://doi.org/10.1145/279232.279236.

Zou, X., and X. Tian, 2018: Hurricane warm-core retrievals from AMSU-A and remapped ATMS measurements with rain 
contamination eliminated. J. Geophys. Res. Atmos., 123, 10 815-10 829, https://doi.org/10.1029/2018JD028934.

- , Y. H. Kuo, and Y. R. Guo, 1995: Assimilation of atmospheric radio refractivity using a nonhydrostatic adjoint model. Mon. Wea. Rev., 123, 2229-2250, https://doi.org/10.1175/1520-0493(1995) $123<2229$ :AOARRU>2.0.CO;2.

-, F. Vandenberghe, M. Pondeca, and Y.-H. Kuo, 1997: Introduction to adjoint techniques and the MM5 adjoint modeling system. NCAR Tech. Note NCAR/TN-435+STR, 110 pp., http://dx.doi.org/10.5065/D6F18WNM.

, H. Liu, J. Derber, J. G. Sela, R. Treadon, I. M. Navon, and B. Wang, 2001: Four-dimensional variational data assimilation with a diabatic version of the NCEP global spectral model: System development and preliminary results. Quart. J. Roy. Meteor. Soc., 127, 1095-1122, https://doi.org/10.1002/ qj. 49712757321. 